\title{
Automatic Number Plate Recognition System
}

\author{
Devesh Khaparde \\ Sinhgad Academy of Engineering \\ Kondhwa, Pune
}

\author{
Heet Detroja \\ Sinhgad Academy of Engineering \\ Kondhwa, Pune
}

\author{
Jainam Shah \\ Sinhgad Academy of Engineering \\ Kondhwa, Pune
}

\author{
Rushikesh Dikey \\ Sinhgad Academy of Engineering \\ Kondhwa, Pune
}

\author{
Bhushan Thakare \\ Sinhgad Academy of Engineering \\ Kondhwa, Pune
}

\begin{abstract}
Automatic number plate recognition (anpr) is a system for identifying the vehicle license plate. It uses image processing technology and ssd model of tensor flow framework along with OCR to locate and recognize a license plate on the vehicle, it also uses knn algorithm to recognize characters in the license plate. The system can be used to train and identify different fonts of license plates as used in India, it can be trained using different datasets of fonts that are used.
\end{abstract}

\section{Keywords}

OCR (Optical Character Recognition), Automatic number plate recognition (ANPR), Single Shot MultiBox Detector (SSD)

\section{INTRODUCTION}

Over the recent period, various technologies consisting of innovative methods to extract vehicle number plate but it proves to be a difficult task. Vehicle proof of identity method can be categorized into four key steps such as license plate location extraction, pre-processing of image, character recognition in the number plate and segmentation of characters. Every single step has its own significance in order to spot the vehicle, when the plate images are slanted and characters are not detectable or wrecked, which makes the separation of each character and recognition very monotonous [4].

Automatic number plate detecting system is an image processing technology used to recognize vehicles by their license number plates. This technology is gaining reputation in security and traffic installations for preserving law enforcement on Civic roads, tool collection, administration of parking lots and stolen cars.

Vehicle licence plate number extraction plays an important part in various applications as follows automatic toll pay, traffic monitoring on highway, identification of stolen vehicles, parking lots access control etc. This kind of emerging protected innovative technology is now used in specific areas, such as military area, Supreme Court, parliament house [7].

In India, there are two forms of license plates, which are used: 1) Black characters with yellow background.

2) Black characters with white background [4].

Recognizing number plates in India is painstaking when equated to foreign license plate because number plates in India may have different fonts and shapes.

\section{METHODOLOGY}

- Capture Frame of vehicle from the CCTV footage

- Input captured frame to the SSD Model.

- Extracting coordinates of bounding box of number plate via SSD model.

- Cropping the number plate for Character recognition

- Input cropped number plate to OCR.

- OCR gives text file of license number plate as output.

- Using text file retrieve details of the vehicle from the database using portal.

\section{STUDIES AND FINDING}

Vehicle number plate detection is very important as it provides security to transportation field. There are many different systems proposed and different techniques used by researchers to detect and recognize plates, by collecting information from various journals and papers formerly done. There were essentially four steps [4] [9] which include processing image as required, number plate localization, segmentation of characters and recognition of characters. For the first step noise removal and conversion from rgb to grey scale is done [8] [3] [4], system to tolerate greater level of noise was proposed [7] to effectively tolerate noise level upto $85 \%$,for some systems first conversion to grey scale and then removal of noise was done, then locating the plate in the image was done using different algorithms, FPGA implementation is used here [1] for this purpose, later character segmentation and recognition is also done with the help of FPGA. Number plates in India do not mostly follow a standard format so it is difficult to build a system which can be applied universally in India hence in [6] various fonts used for number plates in India were taken to be recognized using MATLAB, various templates were formed with different plate fonts and these templates were then compared to fonts of the plates captured for character recognition. In [11] super resolution technique was used for anpr which has the four basic steps mentioned before included. After the recognition of the plate the vehicle can be fined for different violations which [2] shows for parking fee to be collected from the offender. The major purpose of this system is to better the traffic conditions and to help people, [5] paper focuses on optimizing cities traffic signals in smart cities. 


\section{ARCHITECTURE}

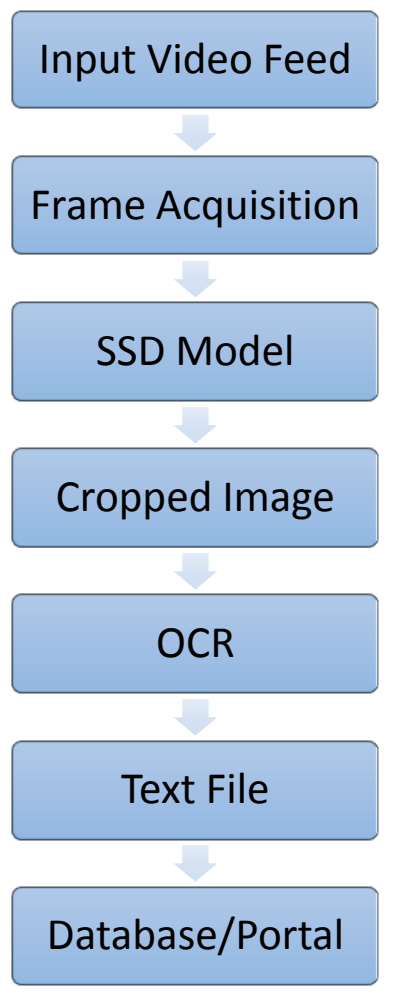

Fig.1 System Architecture

\subsection{SYSTEM OVERVIEW}

The proposed system consists of three modules namely:

- Number Plate Detection using SSD Model.

- Optical Character Recognition.

- Portal for user interface.

\section{A. Number Plate Detection using SSD Model}

This module uses the concept of transfer learning to use pretrained model to train it with custom detector used in the proposed system i.e. license plate detector. With Transfer learning any pre-trained model can then be used as the starting point for a model on the another task of interest so this system uses one of the tensor flow model called Single shot multibox detector as it is best among other high-speed model that can smoothly work with high fps video feed. These module comprises of various steps which are following:

\section{Gathering dataset for training:}

The system required to create and label new custom dataset so different images of cars with license plate were collected and with the help of Image annotation tool namely LabelImg tool it is manually labelled and localized the coordinates of the license plate in that image.

Retrain the model with custom data:

Single Shot Multibox Detector runs a convolutional network on input image only one time to determine a feature map, it also uses anchor boxes at various aspect ratios, and SSD foresees bounding boxes after numerous convolutional layers. Since each convolutional layer functions at an altered scale, it is capable of detecting objects of numerous scales.

Once model is trained with training dataset, it can then be used for predicting bounding box in any new image, after all steps it outputs a cropped image of license plate which is then given to OCR as input for character recognition.

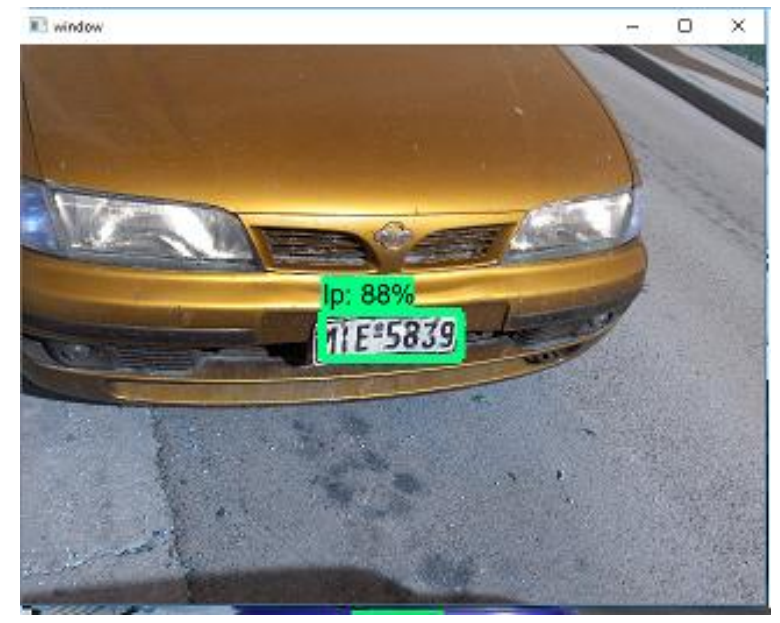

Fig.2 Detected Number Plate 1

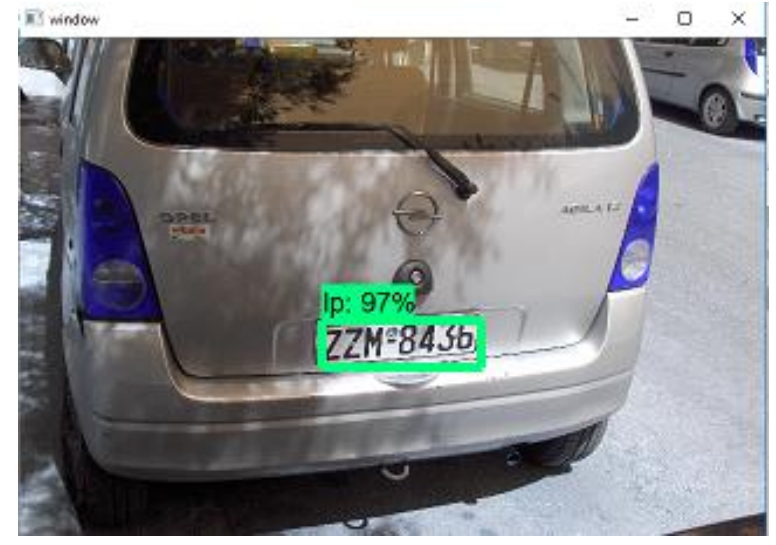

Fig.3 Detected Number Plate 2

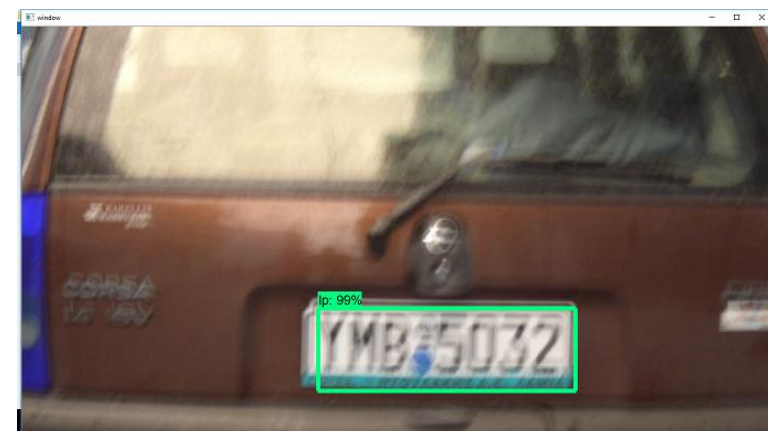

Fig.4 Detected Number Plate 3

\section{B. Optical Character Recognition}

The output of the SSD model will give a cropped image of the license plate from the captured frame. OCR is then used on the cropped image, this module is trained using different datasets of fonts which are used in license plate to recognize the characters on the localized plate, to train the module bounding boxes are created around the each character present in the datasets of fonts one by one and are given keyboard inputs to match the character present in the bounding box, with the help of this two files are created consisting of the Unicode of the characters trained. Finally, knn-algorithm is used to recognize the characters in the plates comparing them with the trained characters. The recognized characters are stored in a text file which can be further used in the portal. 


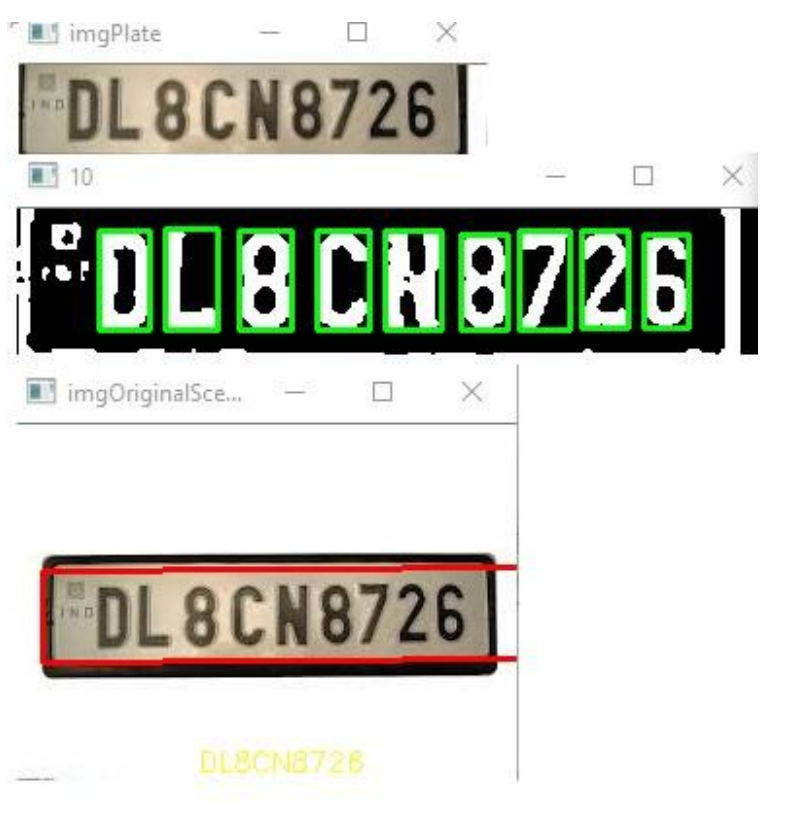

Fig.5 OCR Outputs

\section{Portal for User Interface}

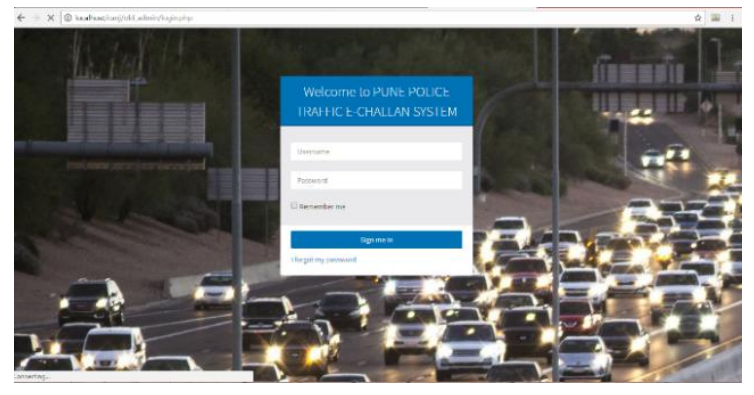

Fig.6 Portal Login Page

The portal will have a login page for all the users and as he $\operatorname{logs}$ in the login id of the user is saved in the system. The user can generate the challan from the portal for the offence that has been done by the vehicle owner/driver. He can directly open the output that has been received from the earlier module into the notepad format and it gets read automatically and is entered into the number plate field and the user can select the offence performed and the user will receive the challan in the form of SMS and Email on the registered Mobile number and Email ID.

\subsection{Result \& Analysis}

\section{A. SSD Model}

The proposed model of the system uses tensorflow framework. The effectiveness of the system was examined by taking 100 different images and calculating their accuracy which came out to be 86.57 $\%$.

\section{B. Optical Character Recognition}

True Positive (TP), True Negative (TN), False Positive (FP) and False Negative (FN) were determined of the test set and were used to calculate the accuracy as:

\section{Accuracy $=(\mathrm{TP}+\mathrm{TN}) /(\mathrm{TP}+\mathrm{TN}+\mathrm{FP}+\mathrm{FN})$}

This gave the accuracy of the system, comparing the values of TP, TN, FP, FN we get the relation between the loss and accuracy as:

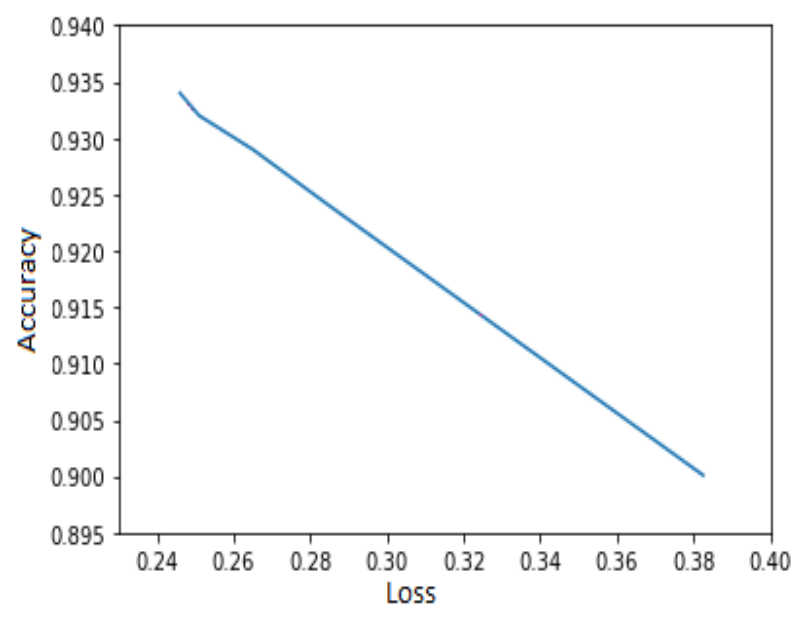

Fig.7 Training Accuracy/Loss Percentage

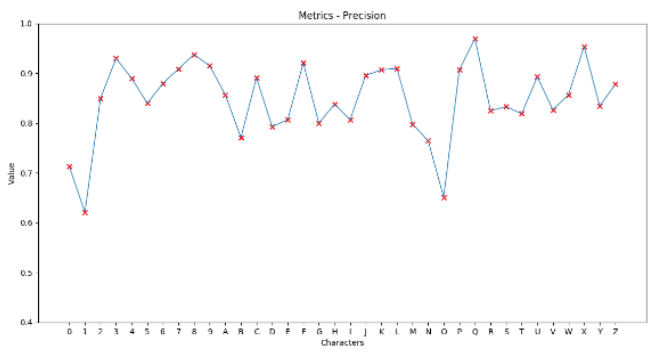

Fig.8 Precision

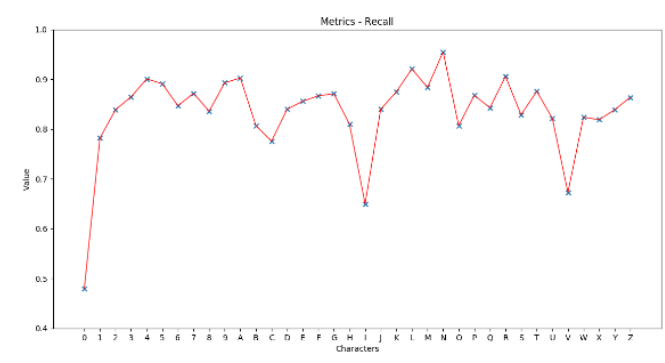

Fig.9 Recall

\section{CONCLUSION}

Hence, we have provided an Automatic License plate Recognition System which uses the concepts of object detection, image processing and machine learning.

For future use the system can be trained further to recognize plates with fancy fonts and plates having different font sizes also the system can be trained with different datasets to recognize plates from different parts of the world. The ssd model can be trained more for better accuracy with more computation power.

\section{REFERENCES}

[1] Yuan Jing, Bahar Youssefi, Mitra Mirhassani, Roberto Muscedere "An Efficient FPGA Implementation of Optical Character Recognition for License Plate Recognition" University of Windsor, ON, Canada,2017 IEEE

[2] Worawut Yimyam, Mahasak Ketcham" The Automated Parking Fee Calculation Using License Plate Recognition 
System” Universitas Gadjah Mada Yogyakarta, Indonesia, 2017 IEEE.

[3] Muayad Ali Hamood Bakhtan, Dr. Munaisyah Abdullah, Dr. Aedah Abd Rahman "A Review on License Plate Recognition System Algorithms”, IEEE 2016.

[4] Mahesh Babu K, M V Raghunadh.” Vehicle Number Plate Detection and Recognition using Bounding Box Method", 2016 IEEE.

[5] Zhiyi Li, Mohammad Shahidehpour, Shay Bahramirad, Amin Khodaei, "Optimizing Traffic Signal Settings in Smart Cities”, IEEE 2015.

[6] J. Albert Mayan, Kumar Akash Deep, Livingston Alvin, Siva Prasad Reddy" Number Plate Recognition using Template Comparison for various fonts in MATLAB", IEEE 2016.

[7] Y. Y. Nguwi W. J. Lim," Number Plate Recognition in Noisy Image",2015

[8] Riazul Islam, Kazi Fatima Sharif, Satyen Biswas, "Automatic Vehicle Number Plate Recognition Using Structured Elements", IEEE 2014.
[9] Amirgaliyev Beibut,Kairanbay Magzhan,Kenshimov Chingiz," Effective Algorithms and Methods for Automatic Number Plate Recognition”,2014 IEEE

[10] Farid Bounini,Denis Gingras,Vincent Lapointe,Herve Pollart," Autonomous Vehicle And Real Time Road Lanes Detection And Tracking", Proceedings of 2015 IEEE

[11]Balamurugan G., Sakthivel Punniakodi, Rajeswari K. and Arulalan V.. "Automatic number plate recognition system using super-resolution technique", 2015 International Conference on Computing and Communications Technologies (ICCCT), 2015.

[12] Sina,A. Wibisono,A. Nurhadiyatna,B. Hardjono,W. Jatmiko," Vehicle Counting and Speed Measurement Using Headlight Detection.”, Proceedings of the 2013 IEEE.

[13] Devesh Khaparde, Heet Detroja, Jainam Shah, Rushikesh Dikey, Bhushan Thakare "Survey On Automatic Number Plate Recognition System”, 2017 International Journal Of Computer Applications (IJCA). 